\title{
Gain-of-function mutations and copy number increases of Notch2 in diffuse large B-cell
}

\section{lymphoma}

Suk-young Lee ${ }^{1}$, Keiki Kumano ${ }^{1,2}$, Kumi Nakazaki ${ }^{2}$, Masashi Sanada ${ }^{1,2}$, Akihiko Matsumoto ${ }^{1}$,

Go Yamamoto $^{2}$, Yasuhito Nannya ${ }^{2}$, Ritsuro Suzuki ${ }^{3}$, Satoshi Ota ${ }^{4}$, Yasunori Ota ${ }^{5}$, Koji Izutsu $^{2}$, Mamiko Sakata-Yanagimoto ${ }^{1,2,8}$, Akira Hangaishi ${ }^{2}$, Hideo Yagita $^{6}$, Masashi Fukayama $^{4}$, Masao Seto ${ }^{3}$, Mineo Kurokawa ${ }^{2}$, Seishi Ogawa ${ }^{1,2,7}$, and Shigeru Chiba ${ }^{1,8}$

${ }^{1}$ Department of Cell Therapy and Transplantation Medicine, University of Tokyo Hospital, Tokyo, Japan; ${ }^{2}$ Department of Hematology and Oncology, Graduate School of Medicine, University of Tokyo, Tokyo, Japan; ${ }^{3}$ Division of Molecular Medicine, Aichi Cancer Center, Nagoya, Japan; ${ }^{4}$ Department of Pathology, Graduate School of Medicine, University of Tokyo, Tokyo, Japan; ${ }^{5}$ Department of Pathology, Toranomon Hospital, Tokyo, Japan; ${ }^{6}$ Department of Immunology, School of Medicine, Juntendo University, Tokyo, Japan; ${ }^{7}$ CREST, Japan Science and Technology Agency, Japan; ${ }^{8}$ Department of Clinical and Experimental Hematology, Graduate School of Comprehensive Human Sciences, University of Tsukuba, Tsukuba, Japan.

Financial support: Grants-in-Aid for Scientific Research from the Ministry of Education, Culture, Sports, Science, and Technology of Japan (KAKENHI \#18013012 and \#19390258); and grants from the Sagawa Foundation for the Promotion of Cancer Research and Osaka Cancer Foundation (SC).

Reprint request should be sent to: Shigeru Chiba, MD, PhD; Department of Hematology, University of Tsukuba, 1-1-1 Tennodai, Tsukuba, Ibaraki 305-8575, Japan. Telephone: 81-29-853-3101. FAX: 81-29-853-8079. E-mail address: schiba-tky@umin.net 
Word count: 3994 words 


\begin{abstract}
Signaling through the Notch1 receptor has a pivotal role in early thymocyte development. Gain of Notch1 function results in the development of T-cell acute lymphoblastic leukemia (T-ALL) in a number of mouse experimental models, and activating Notch1 mutations deregulate Notch1 signaling in the majority of human T-ALLs. Notch2, another member of the Notch gene family, is preferentially expressed in mature B cells and is essential for marginal zone B-cell generation. Here, we report that 5 of 63 ( 8\%) diffuse large B-cell lymphomas (DLBCL), a subtype of mature B-cell lymphomas, have Notch2 mutations. These mutations lead to partial or complete deletion of the PEST domain, or a single amino acid substitution at the C-terminus of Notch2 protein. Furthermore, high-density oligonucleotide microarray analysis revealed that some DLBCL cases also have increased copies of the mutated Notch2 allele. In the Notch activation-sensitive luciferase reporter assay in vitro, mutant Notch2 receptors show increased activity compared with wild-type Notch2. These findings implicate Notch2 gain-of-function mutations in the pathogenesis of a subset of B-cell lymphomas, and suggest broader roles for Notch gene mutations in human cancers.
\end{abstract}




\section{Introduction}

Signaling through the Notch receptor, triggered by the binding of ligands expressed on neighboring cells, has a key role in determining cell fate in a variety of cell lineages, including lymphocytes ${ }^{1,2}$. In mammals, there are four Notch genes that encode structurally similar single-pass and heterodimeric transmembrane receptors. Ligand binding initiates a series of intramolecular cleavages, which eventually liberates the intracellular domain of the transmembrane subunit of the Notch receptor (ICN). The ICN is then translocated to the nucleus and creates a transcriptional activating complex with RBP-J $\kappa$, a constitutive DNA binding protein. During these processes, Notch proteins are intricately regulated by glycosylation, endocytosis, recycling, phosphorylation, and ubiquitylation before and after ICN liberation. Many of these regulatory processes appear to modify the biologic activity of Notch ${ }^{3}$. Notably, polyubiquitylation-based degradation is dependent on the PEST domain, located at the $\mathrm{C}$ terminus of the Notch protein.

The physiologic roles of Notch1 and Notch2 have been clarified in mouse models, particularly in the lymphoid system. Notch1 is preferentially expressed in immature T cells and is essential for specification of early hematopoietic progenitors toward the T-cell fate and for early T-cell development in the thymus ${ }^{4}$. In contrast, Notch2 is preferentially expressed in mature B cells and is required for the generation of a mature B-cell subset, known as splenic marginal zone B (MZB) cells in mice ${ }^{5}$. Notch1 was originally identified as a transforming gene in human T-cell acute lymphoblastic leukemia (T-ALL) cells harboring the $\mathrm{t}(7 ; 9)(\mathrm{q} 34 ; \mathrm{q} 34)$ chromosomal translocation ${ }^{6}$. The N-terminal truncated form of Notch1 expressed in this type of T-ALL cell can induce the development of T-ALL when expressed in bone marrow cells that are then transplanted into recipient mice ${ }^{7}$. Importantly, more than $50 \%$ of childhood and $30 \%$ to $40 \%$ of adult human T-ALL cases carry Notch1 mutations that 
lead to deregulated activation of Notch signaling ${ }^{8-11}$, indicating that accelerated Notch signaling contributes to the development of human neoplasms.

Two regions of the Notch1 gene are major targets of oncogenic mutations in T-ALL. Missense, insertion, and deletion mutations in the heterodimerization domains are thought to decrease the stability of the dimer, consisting of the extracellular and transmembrane subunits, which results in the progression of Notch1 cleavage without ligand stimulation ${ }^{8,12}$. The other series of mutations accumulate in the PEST domain and its N-terminally flanking transactivation domain. All these mutations cause partial or complete deletion of the PEST domain, which are considered to result in the prolonged half-life of Notch1 ICN, because the PEST domain is responsible for polyubiquitylation-based degradation of ICN ${ }^{13}$.

These lines of information about Notch genes led us to examine the possibility that deregulation of Notch2 signaling is involved in the development of mature B-cell lymphomas. We screened Notch2 gene mutations at the heterodimerization and PEST domains in 109 B-cell lymphoma samples, and found mutations in 5 samples, all of which were diffuse large B-cell lymphomas (DLBCL). Interestingly, two of the five samples had an increased copy number of the mutated Notch2 allele, and in another sample of the five the total copy number of the Notch2 allele was increased. Furthermore, we confirmed that the mutation-carrying Notch2 receptors had increased activity when stimulated by a ligand in vitro. We postulate that gain-of-function mutations of Notch2 are involved in the pathogenesis of a subset of DLBCL. 


\section{Materials and methods}

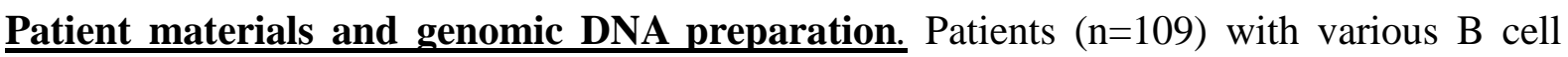
lymphomas were enrolled in the study after informed consent was obtained. The study design was approved by the ethics committees of the University of Tokyo and Aichi Cancer Center. Genomic DNA was extracted from cryopreserved samples using a commercial kit (PUREGENE $^{\mathrm{TM}}$, Gentra, Minneapolis, MN).

PCR-single-strand conformation polymorphism (SSCP). Based on the information of Notch1 mutations in T-ALL and the high similarity between Notch1 and Notch2 genes, we confined our mutation analysis to exons 26, 27, and 35 of Notch2 that correspond to the heterodimerization domains (exons 26 and 27) and the C-terminal region containing the transactivation and PEST domains (exon 34), respectively. Oligonucleotide primers designed to amplify whole exon 26 and exon 27, and five divided portions of exon 35 are listed in Supplemental Table 1 . The ${ }^{32} \mathrm{P}$-labled PCR product was subjected to SSCP analysis as described in the literature ${ }^{14}$. In brief, the PCR mixture was heated at $80^{\circ} \mathrm{C}$ and applied to $5 \%$ polyacrylamide gel containing $10 \%$ glycerol. After 2 - 4 h electrophoresis with cooling, the gel was dried on filter paper and exposed to X-ray film. The PCR products were directly sequenced or bands with aberrant migration were excised from the gel and subjected to direct sequencing when indicated.

High-density oligonucleotide microarray analysis. Genome-wide copy number detection analysis was performed as described previously ${ }^{15}$. In brief, Affimetrix GeneChip Mapping 100 k high-density oligonucleotide arrays (Affimetrix, Inc, Santa Clara, CA) were used and the data were analyzed using the CNAG algorithm.

Fluorescence in situ hybridization. Bacterial artificial chromosome (BAC) clones 
RP11-723d17 (Notch2) and RP11-80d6 (1q23.3) were used to evaluate the copy number of the Notch2 gene. BACs were obtained from the BAC/PAC Resource Center (Children's Hospital, Oakland, CA). Fluorescence in situ hybridization experiments on interphase nuclei were performed as described previously ${ }^{16}$.

Quantitative real-time PCR for genomic DNA. For the copy number evaluation of the Notch2 gene by quantitative real-time PCR, genomic DNA was extracted from samples L8 and W121672; a stomach cancer cell line (MKN45) that had a copy number loss at the Notch2 (1p13) locus (data from microarray analysis not shown); and normal peripheral blood mononuclear cells. The Notch2 gene dosage was measured using the primers: forward, TTCCCCAAGTGAGAGACATTT, and reverse, CAGACACTTCACAGAACAGAA, and normalized by the relative DNA quantities measured by real-time PCR using the control locus (2q35) primers: forward, TGGCTGATGAACTTTTGCAC, and reverse, AGCGGTTGAGGTCTGTGAAC. Student’s t-test was used for the statistical analysis.

Immunohistochemistry. Tissue sections were mounted on silanated slides, deparaffinized with xylene, rehydrated with a series of graded ethanols, processed with an autoclave in 10 mmol/l citrate buffer for 5 min, $\mathrm{pH}$ 6.0, treated with horse serum albumin to block nonspecific staining, and immunostained. The detection of antibody binding was visualized by the avidin biotin complex method using diaminobenzidine as the chromogen. The sections were counterstained with hematoxylin.

Plasmid preparation. In the human full-length Notch2 cDNA (wtN2) (a gift from S. Artavanis-Tsakonas, Harvard University), the stop codon corresponding to the nonsense mutation $(7454 \mathrm{C} / \mathrm{T})$, the single-base deletion mutation corresponding to 7120Del, and the point mutation corresponding to 7614 G/A were introduced. Mutant primers were used for PCR and the resulting products were sequenced and used to replace the corresponding 
fragment of wtN2 cDNA to create the Notch2 with the nonsense mutation and the R2453Q mutation (nsmN2, delstN2, and rqN2, respectively). These cDNAs were inserted in pTracerCMV (Invitrogen, Carlsbad, CA)

\section{Establishment of $\mathrm{CHO}(\mathrm{r})$ cells stably expressing wild-type and mutant human Notch2.}

$\mathrm{CHO}(\mathrm{r})$ cells were transfected with pTracerCMV/wtN2, pTracerCMV/nsmN2, pTracerCMV/delstN2, and pTracerCMV/rqN2, and selected for zeocin (400 $\mu \mathrm{g} / \mathrm{ml})$ resistance. The resulting zeocin-resistant cells were single-cell sorted using the anti-human Notch2 monoclonal antibody (mAb). The anti-human Notch2 (MHN2-25, mouse IgG $_{2 \mathrm{a}}$ ) mAb was generated by immunizing BALB/c mice with human Notch2-Fc (the Fc portion of human $\mathrm{IgG}_{1}$ was fused to the 22nd epidermal growth factor repeat of the extracellular region of human Notch2) and screening hybridomas producing mAbs specific for Notch2-Fc by ELISA. MHN2-25 reacted with $\mathrm{CHO}(\mathrm{r})$ cells expressing human Notch2, as demonstrated by flow cytometry (Supplemental Fig. 1).

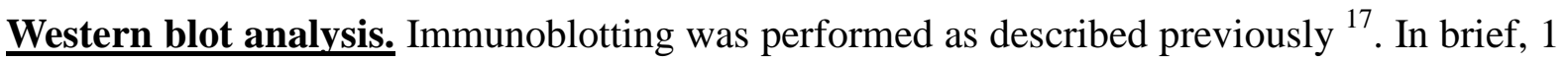
x $10^{6}$ wtN2/CHO(r), nsmN2/CHO(r), delstN2/CHO(r), and rqN2/CHO(r) cells were solubilized in $0.1 \mathrm{ml}$ lysis buffer containing 1\% NP-40, electrophoresed in $7.5 \%$ sodium dodecyl sulfate polyacrylamide gel, transferred onto Immobilon-P membrane (Millipore, Billerica, MA). It was then probed with a monoclonal antibody recognizing the intracellular domain of human and murine Notch2 (C651.6DbHN, University of Iowa Developmental Studies Hybridoma Bank, Iowa City, IA) and an alkaline phosphatase-conjugated secondary antibody (Promega, Madison, WI).

Transcriptional activation assay. The luciferase assay was performed as described previously ${ }^{17}$. In brief, $2 \times 10^{5} \mathrm{CHO}(\mathrm{r})$ cells expressing wtN2, Notch2 with truncatation at 2400 (nsmN2), Notch2 with truncation after 6 amino acids insertion at 2288 (delstN2), and 
Notch2 with an R2453Q mutation (rqN2) were inoculoated in a 6-well dish and the next day transfected with the pGa981-6 luciferase reporter plasmid $(2 \mu \mathrm{g})$ using the Superfect ${ }^{\mathrm{TM}}$ transfection reagent (QIAGEN, Hilden, Germany). The $\beta$-galactosidase-expressing plasmid, pCMV/ $\beta$-Gal $(0.2 \mu \mathrm{g})$ was co-transfected when indicated. The cells were harvested after $3 \mathrm{~h}$, suspended in $3 \mathrm{ml}$ medium, and a $200 \mu \mathrm{l}$ aliquot was re-plated in a 48-well dish coated with soluble human Delta1 (Delta1-Fc, a chimeric protein composed of the extracellular domain of human Delta1 and the Fc portion of human immunoglobulin $\mathrm{G}^{18,19}$, a gift from S. Sakano, Asahi Kasei). After $24 \mathrm{~h}$ incubation, the cellular extracts were used to measure luciferase and, when applied, $\beta$-galactosidase activities. Two independent clones were used to compare the luciferase activity of each Notch2 protein and bulk transfectants were used to evaluate the effect of DAPT (Calbiochem), a $\gamma$-secretase inhibitor. 


\section{Results}

Notch2 gene is mutated in a subset of DLBCL. Notch2 gene mutations were screened in 109 B-cell lymphoma samples, including 63 DLBCLs, 18 follicular lymphomas, and 28 MZB cell lymphomas or mucosa-associated lymphoid tissue lymphomas. Exons 26 and 27, encoding the $\mathrm{N}$ - and $\mathrm{C}$-terminal heterodimerization domains, and a portion of exon 34, encoding the PEST domain and its bilateral flanking regions, were amplified by polymerase chain reaction (PCR) using genomic DNA with the primers listed in Supplemental Table1 and examined for mutations using the single-strand conformational polymorphism (PCR-SSCP) $\operatorname{method}^{20}$.

Five distinct nucleotide changes were detected in 11 of the 109 B-cell lymphoma samples, exclusively in exon 34. Whereas two of the five changes detected in 6 of the 11 samples were single nucleotide polymorphisms (SNP) without amino acid changes, the other 3 nucleotide changes detected in the remaining 5 samples (Fig. 1 A-E) were thought to represent somatic mutations resulting in premature truncation or single amino acid substitution (Table 1). A nonsense mutation, $\mathrm{C}$ to $\mathrm{T}$ at nucleotide 7454 (based on the published human Notch2 sequence, NM_024408), in three cases (Fig. 1A-C) and a single base deletion at position 7120 in another case (Fig. 1D), led to premature truncation of the Notch2 protein (Table 1). These Notch2 proteins lacked a part or the entire region of the PEST domain. The other single nucleotide change, $G$ to $A$ at 7614 , resulted in the replacement of arginine with glutamine on the C-terminal side of the PEST domain (Fig. 1E and Table 1). The G7614A change is not listed in the public SNP database (http://www.ncbi.nlm.nih.gov/projects/SNP/; as of October 23, 2007). In addition, the dose of the mutant A allele was unbalanced relative to the wild-type $G$ allele (Fig. 1E), further decreasing the possibility of an SNP. Constitutive DNA was available in one case (W109539) 
and was confirmed to be the wild-type sequence (Fig. 1F), which definitely concluded that the mutation in the tumor was somatic origin.

Mutation-carrying cases show the same expression pattern of CD10, BCL6, and MUM-1. All five cases with Notch2 mutations were diagnosed as DLBCL, and were uniformly immunohistochemically negative for CD10 and positive for BCL6 and MUM-1 (Fig. 2). We have reviewed 24 DLBCL subjects without Notch2 mutations for expression of CD10, BCL6 and MUM-1. The immunohistochemistry study revealed that CD10, BCL6, and MUM-1 were positive in 4, 19, and 16 subjects, respectively. Among these, the CD10-negative, BCL6-positive, and MUM-1-positive staining pattern was seen in 10 (data not shown). Thus, this pattern was seen in five out of five Notch2 mutation-carrying subjects and 10 out of 24 Notch2 mutation-negative subjects, making the comparison statistically significant ( $\mathrm{p}=0.042$; Fisher's exact test). This estimation is consistent with the previous report ${ }^{21}$ and indicates that CD10-negative, BCL6-positive, and MUM-1-positive DLBCL might represent a fraction of non-germinal center B-cell-like DLBCL (non-GCB-DLBCL), according to the immunohistchemistry-based DLBCL subclassification ${ }^{21}$. DLBCL cases carrying the gain-of-function-type Notch2 mutations, thus, might constitute a discrete subset of non-GCB-DLBCL.

\section{Some mutation-carrying samples have an increased copy number of the mutated Notch2}

allele. Of particular interest is the fact that some oncogenic mutations are associated with increases in DNA copy number ${ }^{22,23}$. A high-density oligonucleotide microarray analysis ${ }^{15}$ was performed for 35 among 63 DLBCL samples in the current cohort to evaluate genome-wide copy number alterations. This analysis revealed an increased copy number of the Notch2 allele in the two samples, both of which carried the nonsense mutation. The other 33 samples did not show Notch2 copy number alterations. In one sample (W109539), 
amplification of the Notch2 locus in chromosome 1p was demonstrated by microarray (Fig. 3A, left panel) and fluorescence in situ hybridization (Fig. 3B) analyses. An allele-specific copy number detection analysis revealed an increase in the copy number of a single Notch2 allele (Fig. 3A, left panel). This allele must correspond to the allele carrying the mutated Notch2 gene because the mutated band was overwhelmingly dominant in the PCR-SSCP analysis (Fig. 1A). In the other sample (W121672) with a Notch2 copy number increase, the genomic region encompassing the Notch2 locus on chromosome 1p through the telomere of chromosome 1q had three copies, whereas most of the 1p region had only one copy (Fig. 3A, right panel). The Notch2 copy number increase was confirmed by a quantitative real-time PCR analysis (Fig. 3C). We were unable to determine whether the third Notch2 allele contained wild-type or mutant Notch2 in this sample. In the third sample carrying the nonsense mutation (L8), a change in the Notch2 copy number was not detected in the microarray analysis (data not shown) and quantitative PCR analysis revealed that the copy number was normal (Fig. 3C). Both Notch2 alleles in this sample, however, were likely to have the nonsense mutation, thus representing uniparental disomy, losing the wild-type Notch2, because the mutant band was overwhelmingly dominant in the PCR-SSCP analysis (Fig. 1C). Taken together, these findings indicate that some DLBCL samples have Notch2 mutations and an increased copy number of the mutated Notch2 gene.

Notch2 receptors with mutations have increased activity in vitro. To investigate the function of the Notch2 receptors encoded by mRNA with the nonsense mutation (nsmN2), the single-base deletion mutation (delst), and missense mutation (rqN2), we established CHO(r) cell lines ${ }^{17}$ expressing wild-type Notch2, nsmN2, delstN2, and rqN2 [wtN2/CHO(r), nsmN2/CHO(r), delstN2/CHO(r), and rqN2/ $\mathrm{CHO}(\mathrm{r})]$ and obtained independent clones expressing each Notch2 protein at similar levels, using fluorescence-activated cell sorting with human Notch2-specific antibody (Fig. 4A; Supplemental Fig. 1). A Western blot analysis 
demonstrated that the expected sizes of the transmembrane subunit species were expressed at comparable levels (Fig. 4B). In a Notch-sensitive luciferase reporter assay ${ }^{24}$, the luciferase activity was significantly increased in nsmN2/CHO(r), delstN2/CHO(r), and rqN2/CHO(r) cells, compared with that in wtN2/CHO(r) cells when stimulated with Delta1-Fc. Basal luciferase activities with control IgG also tended to be higher in the three mutant Notch2-expressing $\mathrm{CHO}(\mathrm{r})$ cells lines than that in wtN2/CHO(r) (Fig. 4C). These results indicated that all the three kinds of mutation-carrying Notch2 had significantly increased levels of transcriptional activity compared with wtN2, irrespective of the strength of the Delta1 stimulation.

To evaluate the effect of $\gamma$-secretase inhibitor on wtN2 and nsmN2, we added graded concentrations of DAPT to the Delta1-Fc-stimulated bulk wtN2/CHO(r) and nsmN2/CHO(r). The elevated luciferase activity was reproducible with the bulk nsmN2/CHO(r), which was reduced by DAPT in a concentration-dependent manner (Fig. 4D). The luciferase levels of both wtN2/CHO(r) and nsmN2/CHO(r) at $3 \mu \mathrm{M}$ DAPT in the presence of Delta1-FC were below those in the presence of control IgG without DAPT, respectively, implying the spontaneous Notch2 activity with only IgG in the culture system. The results also indicate that the increased Notch2 activity by the PEST domain deletion is still dependent on the $\gamma$-secretase activity. 


\section{Discussion}

The results of the present study demonstrated gain-of-function mutations of Notch2 and increased copy numbers of the mutated Notch2 allele in a subset of DLBCL. Both nonsense mutations and single base deletion mutations that we found in Notch2 cause partial or complete deletion of the Notch2 PEST domain. Given the marked structural similarities between Notch1 and Notch2, these mutations are thought to prolong the half-life of Notch2 ICN. In some T-ALL cell lines, both heterodimerization and PEST domain mutations lie in cis in the same Notch1 allele. The reporter transcriptional activity of Notch1 with these double mutations was remarkably higher than that of wild-type Notch1 and Notch1 with a single mutation at either the heterodimerization or PEST domain in the absence of exogenous ligand stimulation. The activity of Notch1 with a PEST domain deletion mutation alone was only marginally higher than that of wild-type Notch $1^{8}$. We did not detect mutations in either heterodimerization domain of Notch2 in the current cohort. It might be possible to identify those mutations if the number of samples is increased. With the PEST domain deletion alone, however, nsmN2 had a highly significant increase in activity compared with wtN2. Thus, there appears to be some disagreement between the effects of Notch1 PEST domain deletion and Notch2 PEST domain deletion, although difference in the experimental systems used in the two studies might cause such apparent disagreement. It remains to be determined whether similar mutations found in Notch1 and Notch2 have different biochemical and biologic significances.

The activity was also increased in the rqN2, which has the 2453R/Q single amino acid substitution. This amino acid is located on the C-terminal side of the PEST domain, and it is unknown whether this change affects the structure or function of the PEST domain. Nevertheless, as the arginine residue is often a target of protein modification such as 
methylation ${ }^{25,26}$, this amino acid change might convey a significant alteration in the protein function and be involved in lymphomagenesis.

There are other examples of copy number increases associated with oncogenic gene alterations, such as double Philadelphia chromosomes (BCR/ABL copy number increase) in the blastic crisis of chronic myelogenous leukemia ${ }^{27}$ and homozygous JAK2 mutations in polycythemia vera ${ }^{22,23}$, both of which represent clonal evolution and selection. In the present study, we showed that at least two (or possibly three) cases had increased copy numbers of the mutated Notch2 allele due to gene amplification or mitotic recombination. This finding agrees with the recent understanding that the allelic copy number increases after an oncogenic mutation are a common mechanism of further transformation and selection of neoplastic cells.

Whether the presence of Notch2 gain-of-function mutations has a prognostic indicator or further define a clinical entity within DLBCL is yet to be clarified in future. Although the number of cases are still smalll, our finding that all five cases with Notch2 mutations showed the same immunohistochemical staining pattern for CD10, BCL6, and MUM-1 might provide insight into this issue. DLBCL is highly heterogeneous clinically, morphologically, and genetically. The tissue microarray study based on immunostaining of the tissue samples identified three antigens (CD10, BCL6, and MUM-1) as useful markers to predict the results of mRNA expression array studies ${ }^{28-30}$, and the staining pattern of these three antigens could be used to divide DLBCL cases into germinal center B-cell-like (GCB) and non-GCB groups ${ }^{21}$. Whereas all the five cases carrying Notch2 mutations in our study belonged to the non-GCB group of DLBCL in this criterion, Troen, et al. have recently reported Notch2 mutations in two cases of MZB cell lymphomas ${ }^{31}$. Positions of these mutations are different from those that we demonstrated, and their effect on the Notch2 
function is not demonstrated. We did not find Notch2 mutations in MZB cell lymphomas in our cohort, yet the number of samples was not sufficient to draw conclusions. Although we were unable to find evidence that some or all the five cases carrying Notch2 mutations in our cohort are DLBCL transformed from MZB cell lymphoma, this might be an interesting possibility.

Enhanced activation of Notch signaling by exogenous ligand stimulation or expression of constitutively active Notch proteins supports the growth of a variety of tumor cells, including chronic lymphocytic leukemia ${ }^{32}$, non-Hodgkin lymphoma, and multiple myeloma ${ }^{33}$ cells. Alternatively, inhibition of Notch signaling by $\gamma$-secretase inhibitors suppresses the growth of those tumor cells, in which enhanced Notch signaling might be involved in tumorigenesis ${ }^{34}$. In contrast, a study of mice with a Notch1 deletion in keratinocytes revealed the tumor-suppressive feature of Notch signaling ${ }^{35}$. In a similar context, Notch2 activation induces growth suppression in a wide range of B cell malignancies, raising the possibility that Notch2 functions as a tumor suppressor in B cells ${ }^{36}$. Thus, there appears to be a controversy regarding whether Notch signaling has an oncogenic or anti-oncogenic role in mature B cell malignancies. It might be possible that Notch signaling can induce both growth suppression and tumor promotion in the B cell compartment, depending on the target window within the various developmental stages of B cells.

Although sledom lamina fo tnempoleved gnidulcni seiduts lanoitidda eriuqer lliw ti sisenegamohpmyl ni snoitatum 2 hctoN eht rof elor eht tuoba noisulcnoc evitinifed a ward ot, our observations in this study strongly indicate that deregulation of Notch2 signaling by somatic Notch2 gene abnormalities contributes to the development of a subset of DLBCL, the most frequent type of non-Hodgkin lymphoma. Developing inhibitors of individual Notch molecules, therefore, might provide a new strategy for the treatment of different kinds of 
malignancies, including T-ALL and DLBCL. 


\section{Acknowledgments}

We thank Dr. S. Sakano (Asahi Kasei Co.) for the human Delta1 cDNA, Dr. S. Shirahata (Kyushu University) for CHO(r), Dr. S. Artavanis-Tsakonas (Harvard University) for the human full-length Notch2 cDNA, and Dr. Harashima (Hayashibara Biomedical

Institute) for the cell lines. We are grateful for the support provided by Dr. M. Kato (University of Tokyo) and technical assistance by Y. Mori. 


\section{References}

[1] Chiba S. Notch signaling in stem cell systems. Stem Cells. 2006; 24: 2437-47.

[2] Wilson A, Radtke F. Multiple functions of Notch signaling in self-renewing organs and cancer. FEBS Lett. 2006; 580: 2860-8.

[3] Bray SJ. Notch signalling: a simple pathway becomes complex. Nat Rev Mol Cell Biol. 2006; 7: 678-89.

[4] Radtke F, Wilson A, Stark G, et al. Deficient T cell fate specification in mice with an induced inactivation of Notch1. Immunity. 1999; 10: 547-58.

[5] Saito T, Chiba S, Ichikawa M, et al. Notch2 is preferentially expressed in mature B cells and indispensable for marginal zone B lineage development. Immunity. 2003; 18: 675-85.

[6] Ellisen LW, Bird J, West DC, et al. TAN-1, the human homolog of the Drosophila notch gene, is broken by chromosomal translocations in T lymphoblastic neoplasms. Cell. 1991; 66: 649-61.

[7] Pear WS, Aster JC, Scott ML, et al. Exclusive development of T cell neoplasms in mice transplanted with bone marrow expressing activated Notch alleles. J Exp Med. 1996; 183: 2283-91.

[8] Weng AP, Ferrando AA, Lee W, et al. Activating mutations of NOTCH1 in human T cell acute lymphoblastic leukemia. Science. 2004; 306: 269-71.

[9] Lee SY, Kumano K, Masuda S, et al. Mutations of the Notch1 gene in T-cell acute lymphoblastic leukemia: analysis in adults and children. Leukemia. 2005; 19: 1841-3.

[10] Zhu YM, Zhao WL, Fu JF, et al. NOTCH1 mutations in T-cell acute lymphoblastic leukemia: prognostic significance and implication in multifactorial leukemogenesis. Clin Cancer Res. 2006; 12: 3043-9.

[11] Breit S, Stanulla M, Flohr T, et al. Activating NOTCH1 mutations predict favorable 
early treatment response and long-term outcome in childhood precursor T-cell lymphoblastic leukemia. Blood. 2006; 108: 1151-7.

[12] Sanchez-Irizarry C, Carpenter AC, Weng AP, Pear WS, Aster JC, Blacklow SC. Notch subunit heterodimerization and prevention of ligand-independent proteolytic activation depend, respectively, on a novel domain and the LNR repeats. Mol Cell Biol. 2004; 24: 9265-73.

[13] Oberg C, Li J, Pauley A, Wolf E, Gurney M, Lendahl U. The Notch intracellular domain is ubiquitinated and negatively regulated by the mammalian Sel-10 homolog. J Biol Chem. 2001; 276: 35847-53.

[14] Murakami Y, Hayashi K, Hirohashi S, Sekiya T. Aberrations of the tumor suppressor p53 and retinoblastoma genes in human hepatocellular carcinomas. Cancer Res. 1991; 51: 5520-5.

[15] Nannya Y, Sanada M, Nakazaki K, et al. A robust algorithm for copy number detection using high-density oligonucleotide single nucleotide polymorphism genotyping arrays. Cancer Res. 2005; 65: 6071-9.

[16] Wang L, Ogawa S, Hangaishi A, et al. Molecular characterization of the recurrent unbalanced translocation der(1;7)(q10;p10). Blood. 2003; 102: 2597-604.

[17] Shimizu K, Chiba S, Kumano K, et al. Mouse jagged1 physically interacts with notch2 and other notch receptors. Assessment by quantitative methods. J Biol Chem. 1999; 274: 32961-9.

[18] Karanu FN, Murdoch B, Miyabayashi T, et al. Human homologues of Delta-1 and Delta-4 function as mitogenic regulators of primitive human hematopoietic cells. Blood. 2001; 97: 1960-7.

[19] Suzuki T, Yokoyama Y, Kumano K, et al. Highly efficient ex vivo expansion of human hematopoietic stem cells using Delta1-Fc chimeric protein. Stem Cells. 2006; 24: 
2456-65.

[20] Hangaishi A, Ogawa S, Imamura N, et al. Inactivation of multiple tumor-suppressor genes involved in negative regulation of the cell cycle, MTS1/p16INK4A/CDKN2, MTS2/p15INK4B, p53, and Rb genes in primary lymphoid malignancies. Blood. 1996; 87: 4949-58.

[21] Hans CP, Weisenburger DD, Greiner TC, et al. Confirmation of the molecular classification of diffuse large B-cell lymphoma by immunohistochemistry using a tissue microarray. Blood. 2004; 103: 275-82.

[22] James C, Ugo V, Le Couedic JP, et al. A unique clonal JAK2 mutation leading to constitutive signalling causes polycythaemia vera. Nature. 2005; 434: 1144-8.

[23] Kralovics R, Passamonti F, Buser AS, et al. A gain-of-function mutation of JAK2 in myeloproliferative disorders. N Engl J Med. 2005; 352: 1779-90.

[24] Shimizu K, Chiba S, Hosoya N, et al. Binding of Delta1, Jagged1, and Jagged2 to Notch2 rapidly induces cleavage, nuclear translocation, and hyperphosphorylation of Notch2. Mol Cell Biol. 2000; 20: 6913-22.

[25] Bedford MT, Richard S. Arginine methylation an emerging regulator of protein function. Mol Cell. 2005; 18: 263-72.

[26] Blanchet F, Cardona A, Letimier FA, Hershfield MS, Acuto O. CD28 costimulatory signal induces protein arginine methylation in T cells. J Exp Med. 2005; 202: 371-7.

[27] Avanzi GC, Giovinazzo B, Saglio G, et al. Duplication of $\mathrm{Ph}$ and of 9q+ chromosomes during the blastic transformation of a CML case. Cancer Genet Cytogenet. 1987; 29: 57-63.

[28] Alizadeh AA, Eisen MB, Davis RE, et al. Distinct types of diffuse large B-cell lymphoma identified by gene expression profiling. Nature. 2000; 403: 503-11.

[29] Shipp MA, Ross KN, Tamayo P, et al. Diffuse large B-cell lymphoma outcome 
prediction by gene-expression profiling and supervised machine learning. Nat Med. 2002; 8: 68-74.

[30] Rosenwald A, Wright G, Chan WC, et al. The use of molecular profiling to predict survival after chemotherapy for diffuse large-B-cell lymphoma. N Engl J Med. 2002; 346: 1937-47.

[31] Troen G, Wlodarska I, Warsame A, Hernandez Llodra S, De Wolf-Peeters C, Delabie J. NOTCH2 mutations in marginal zone lymphoma. Haematologica. 2008; 93: 1107-9.

[32] Hubmann R, Schwarzmeier JD, Shehata $M$, et al. Notch2 is involved in the overexpression of CD23 in B-cell chronic lymphocytic leukemia. Blood. 2002; 99: 3742-7.

[33] Jundt F, Probsting KS, Anagnostopoulos I, et al. Jagged1-induced Notch signaling drives proliferation of multiple myeloma cells. Blood. 2004; 103: 3511-5.

[34] Leong KG, Karsan A. Recent insights into the role of Notch signaling in tumorigenesis. Blood. 2006; 107: 2223-33.

[35] Nicolas M, Wolfer A, Raj K, et al. Notch1 functions as a tumor suppressor in mouse skin. Nat Genet. 2003; 33: 416-21.

[36] Zweidler-McKay PA, He Y, Xu L, et al. Notch signaling is a potent inducer of growth arrest and apoptosis in a wide range of B-cell malignancies. Blood. 2005; 106: 3898-906. 


\section{Figure legends}

Figure 1. Mutations of the Notch2 gene in DLBCL. PCR-SSCP and sequence analyses for samples having the nonsense mutation at 7454, C/T (W109539, W121672, L8). Arrowheads indicate shifted bands. The shifted bands in (A) and (C) are obviously dominant against the normal band, suggesting the small amount of normal tissue contamination and unbalanced ratio of mutant and normal alleles. Those in (B) and (E) are minor compared with the normal band, suggesting the contamination of normal tissues, and those in (C) are comparable with the normal band. The shifted bands were excised from the gel and the extracted DNA was sequenced for samples W121672 and W117336. (D) Sequence of DNA prepared from the bone marrow cells obtained from the patient W109539.

Figure 2. Immunohistochemical staining of lymphoma specimens for CD10, BCL6, and MUM1. Antibodies used were anti-CD10 monoclonal antibody (56C6, Novocastra, Norwell, MA), anti-BCL6 monoclonal antibody (P1F6, Novocastra), and anti-human MUM1 monoclonal antibody (MUM1p, Dako, Glostrup, Denmark). The detection of antibody binding was visualized by the avidin biotin complex method using diaminobenzidine as the chromogen. Microscope, Elipse 80i (Nikon). Original magnification, x200. Camera, Dxm1200F (Nikon). Acquisition software, Act-1 (Nikon).

Figure 3. Copy number increases of mutated Notch2 allele in DLBCL. (A) High-density oligonucleotide microarray analysis using the CNAG program for samples W109539 and W121672. The copy number of the Notch2-encompassing allele is greatly increased in W109539 and mildly increased in W121672. Red arrow, centromere. (B) Fluorescence in situ hybridization analysis for sample W109539 using probes corresponding to Notch2 (green signals) and a reference sequence on 1q23.3 (red signals). (C) Copy number evaluation of the Notch2 gene by quantitative real-time PCR for samples L8 and W121672. The quantity of 
genomic DNA, extracted from samples L8 and W121672; MKN45 [a stomach cancer cell line having a copy number loss at the Notch2 (1p13) locus]; and normal peripheral blood mononuclear cells (PBMNC), was normalized by real-time RT-PCR for the control locus (2q35). Statistical analysis (Student's t-test) demonstrated that the Notch2 gene dose was unchanged in sample L8, and significantly increased in sample W121782, relative to the Notch2 gene dose in the PBMNC, whose mean level was adjusted to two copies. *, $\mathrm{p}<0.0001$; **, $\mathrm{p}=0.79$. The number of samples, 24 in each arm.

Figure 4. Functional analysis of wtN2, nsmN2, delstN2, and rqN2. (A) Flow cytometric analysis of $\mathrm{CHO}(\mathrm{r})$ clones expressing wtN2, nsmN2, delstN2, and rqN2 at similar expression levels. Each clone (cl1 and $\mathrm{cl} 2$ represented by green and red lines, respectively) of wtN2/CHO(r), nsmN2/CHO(r), delstN2, and rqN2/CHO(r) was analyzed by flow cytometry using the anti-human Notch2 antibody MHN2-25 (see Supplemental Fig. 1). Purple curves represent isotype control. (B) Western blot analysis of $\mathrm{CHO}(\mathrm{r})$ clones expressing wtN2, nsmN2, delstN2, and rqN2 using an antibody recognizing the intracellular domain of Notch2. Asterisks indicate the transmembrane species of each Notch2 protein. (C) Reporter gene transactivation by wtN2, nsmN2, delstN2, and rqN2. Each clone (cl1 and cl2) was cultured in a dish coated with human Delta1-Fc (D1-FC) or control immunoglobulin G (IgG). Data are means of quadricate experiments. Error bars represent standard deviations. A representative experiment from repeated experiments is shown. sRAU, relative arbitrary units standardized by $\beta$-galctosidase activity. (D) Inhibition of luciferase activity by DAPT, a $\gamma$-secretase inhibitor. Bulk CHO(r) cells transfected with wtN2 or nsmN2 were stimulated with D1-Fc or control IgG with graded concentration of DAPT.

List of supplementary materials: Supplementary Table1 and Supplementary Figure1 
Table 1 Characteristics of five patients who had Notch2 mutations

\begin{tabular}{|c|c|c|c|c|c|}
\hline Patient & Age/sex & CS/IPI* & Treatment/Response & Survival & Others \\
\hline W109539 & $64 / \mathrm{M}$ & IIIA/LI $\dagger$ & R-CHOP $\ddagger / C R u \S /$ relapse & 1.6 y (d) \| & $\begin{array}{c}\text { acromegaly, } \\
\text { DM, AAA** } \\
\text { (post-operation) }\end{array}$ \\
\hline W121672 & $71 / \mathrm{M}$ & $\mathrm{NA} \dagger \dagger$ & NA & NA & - \\
\hline L8 & 66/M & IVA/NA & NA & NA & - \\
\hline $\mathrm{L} 2$ & $61 / \mathrm{F}$ & IV/NA & $\mathrm{CHOP} / \mathrm{CR}$ & 7 y (alive) & $\begin{array}{c}\mathrm{BCL} 2 \\
\text { rearrangement }\end{array}$ \\
\hline W117336 & $83 / F$ & IIIA/LI & $\mathrm{RT}, \mathrm{CHOP}^{* * *}$ & $0.3 \mathrm{y}(\mathrm{d}) * * *$ & - \\
\hline
\end{tabular}

*clinical stage/international prognostic index.

†low intermediate.

$\ddagger$ THP-adriamycin instead of adriamicin, 4 courses.

§complete remission uncertain

|died of advanced lymphoma

**diabetes mellitus, abdominal aortic aneurysm

††information not available

***died of advanced lymphoma after 1st chemotherapy

Table 2 Notch2 mutational status in DLBCL

\begin{tabular}{cccccccc}
\hline Sample & Disease & $\begin{array}{c}\text { Nucleic acid } \\
\text { change }\end{array}$ & $\begin{array}{c}\text { Amino acid } \\
\text { change }\end{array}$ & Copy number & \multicolumn{2}{c}{ Immunohistochemistry } \\
\cline { 6 - 8 } & & CD10 & BCL6 & MUM1 \\
\hline W109539 & DLBCL & $7454 \mathrm{C} / \mathrm{T}$ & 2400 Stop & multiple & - & + & + \\
W121672 & DLBCL & $7454 \mathrm{C} / \mathrm{T}$ & $2400 \mathrm{Stop}$ & 3 & - & + & + \\
L8 & DLBCL & $7454 \mathrm{C} / \mathrm{T}$ & $2400 \mathrm{Stop}$ & $2 *$ & - & + & + \\
L2 & DLBCL & $7120 \mathrm{Del} \mathrm{A}$ & 2288PLKGSTStop & NA & - & + & + \\
W117336 & DLBCL & $7614 \mathrm{G} / \mathrm{A}$ & 2453 R/Q & 2 & - & + & + \\
\hline
\end{tabular}

*uniparental disomy for the mutated Notch2 allele is indicated. 
Fig. 1

A

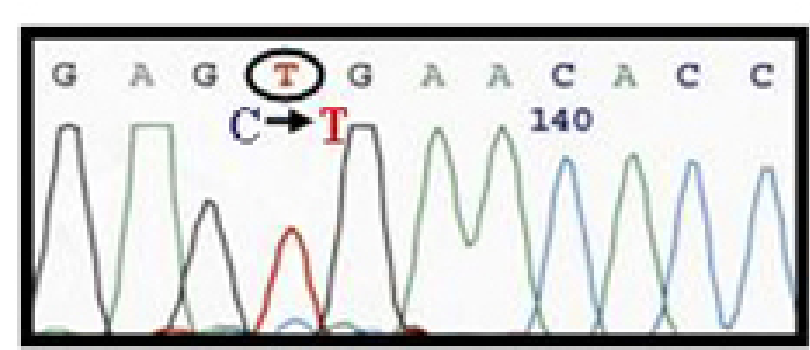

W109539

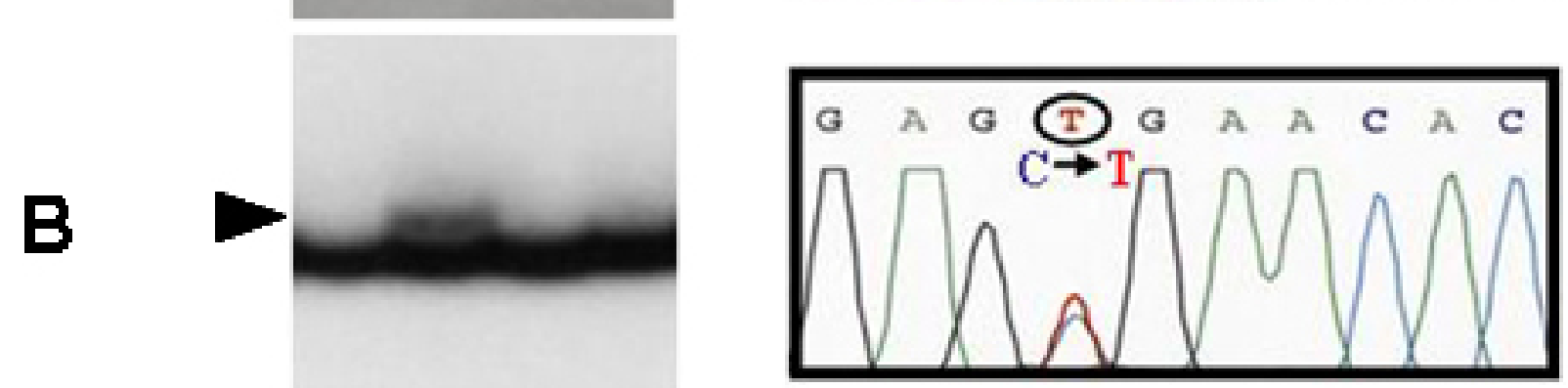

W121672
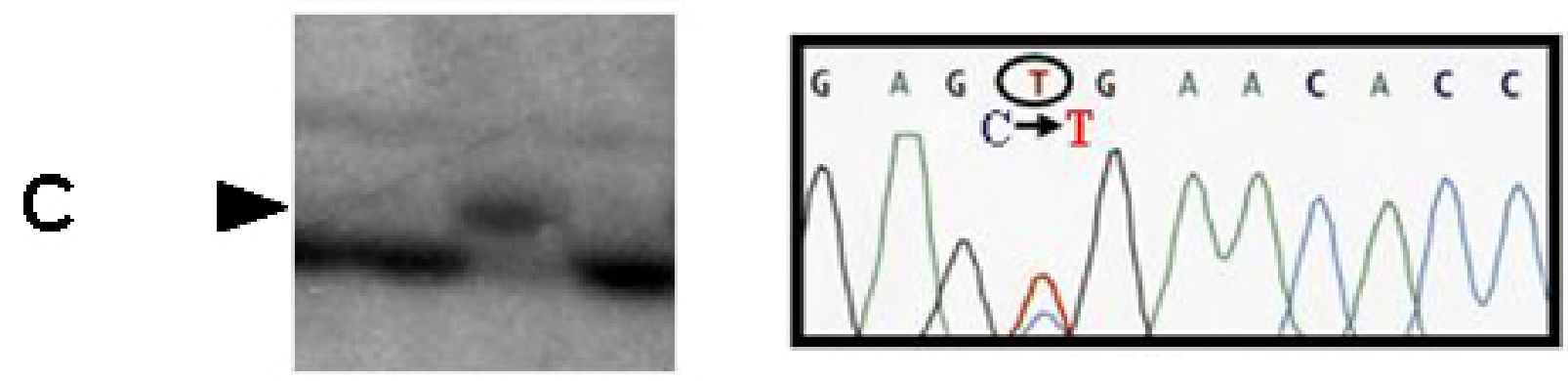

L8
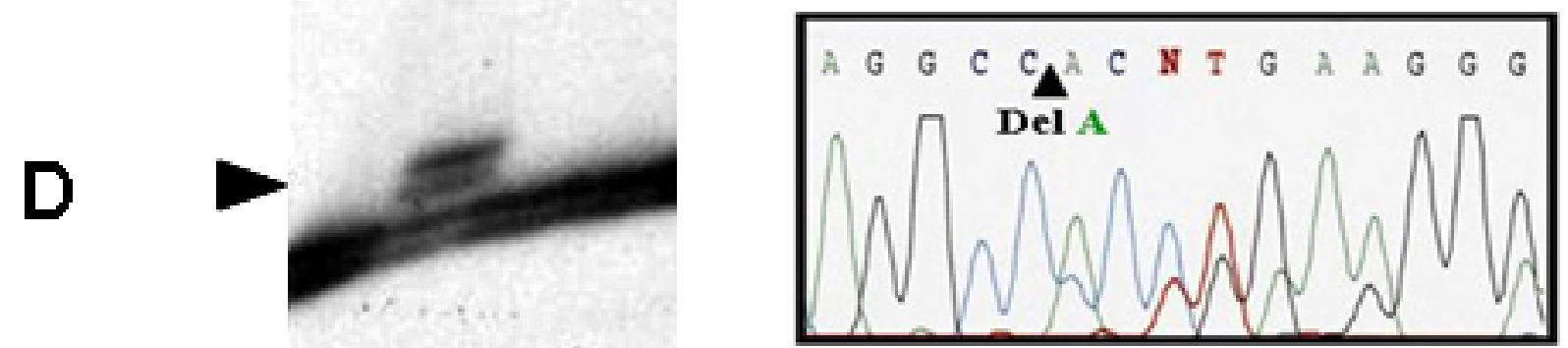

L2

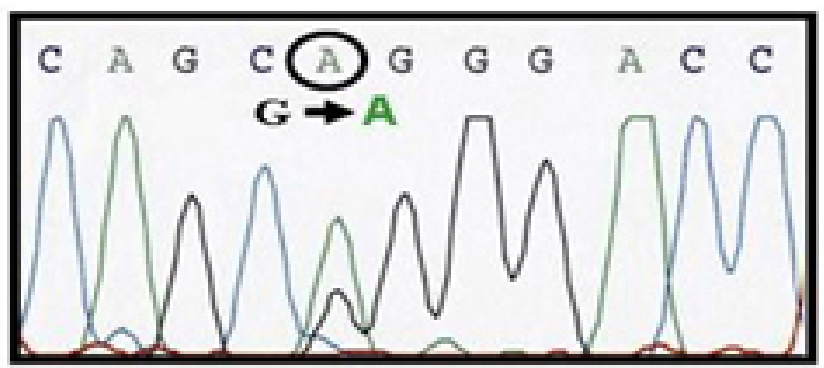

W117336

E

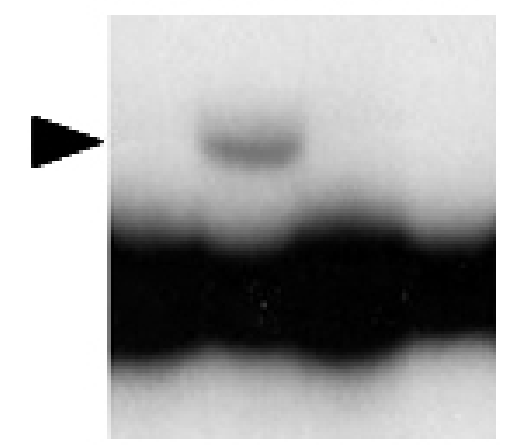

F

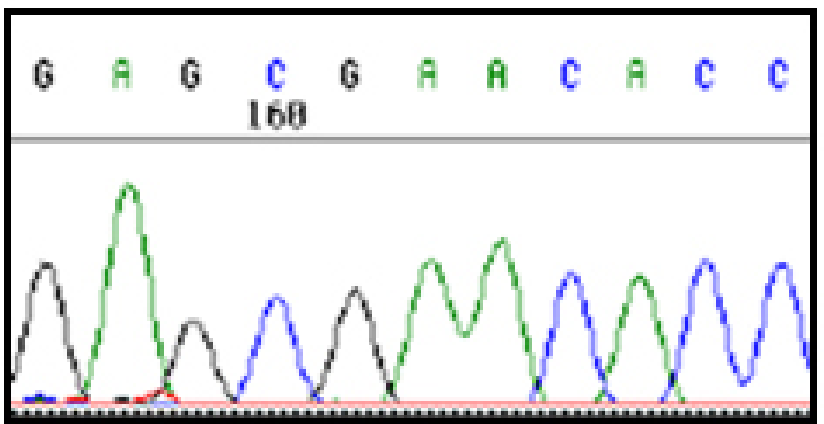

W109539

(bone marrow) 
Fig. 2

CD10

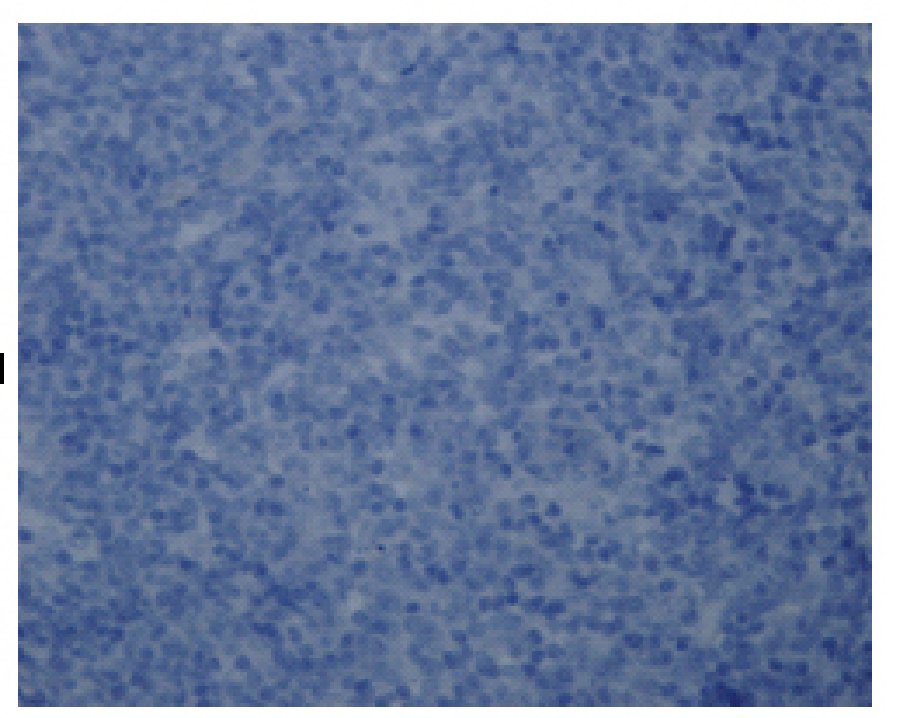

W109539

W121672

W117336
BCL6
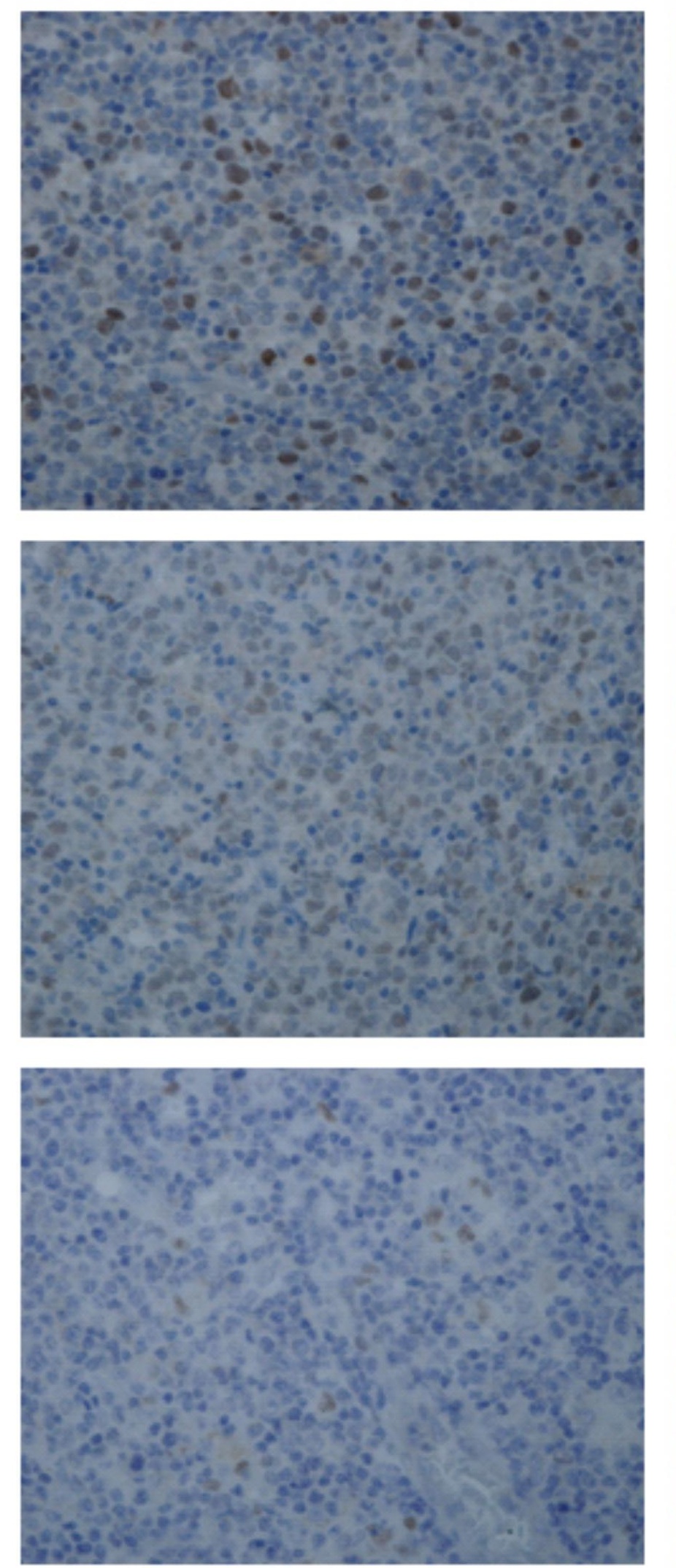

MUM1
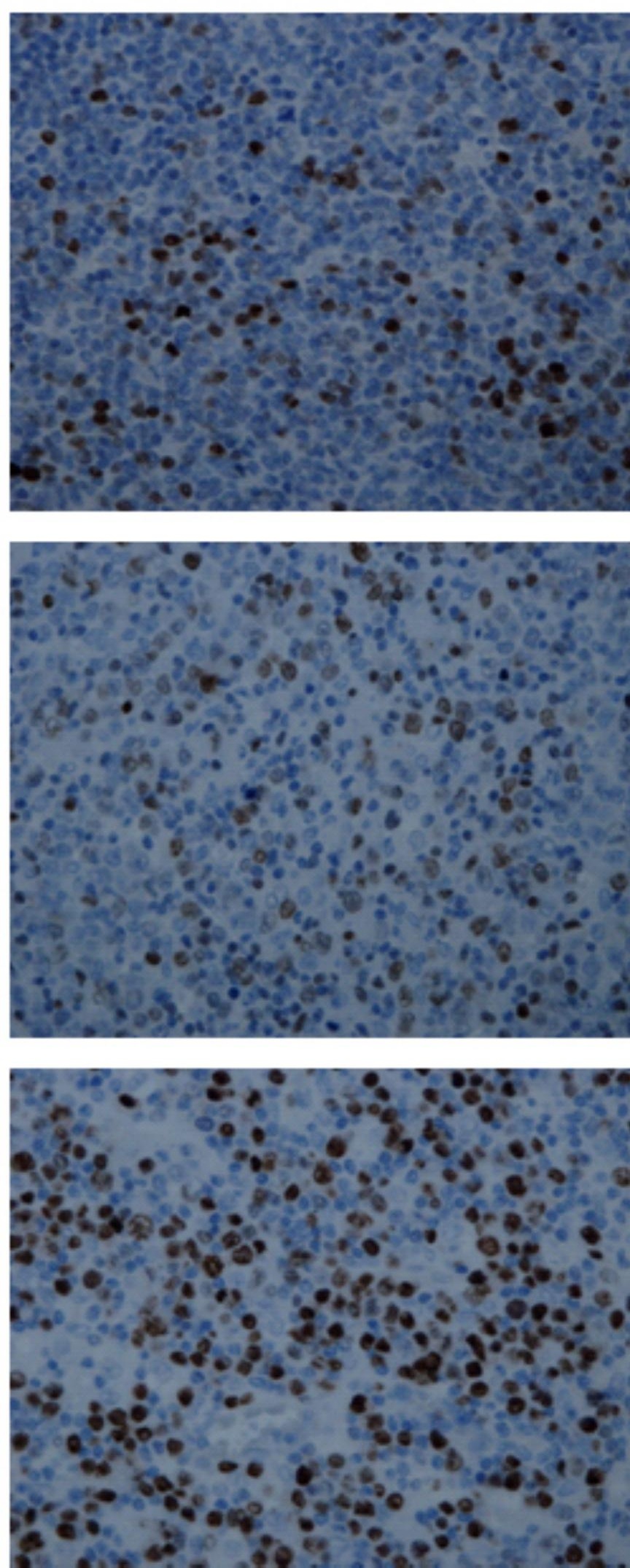
Fig. 3

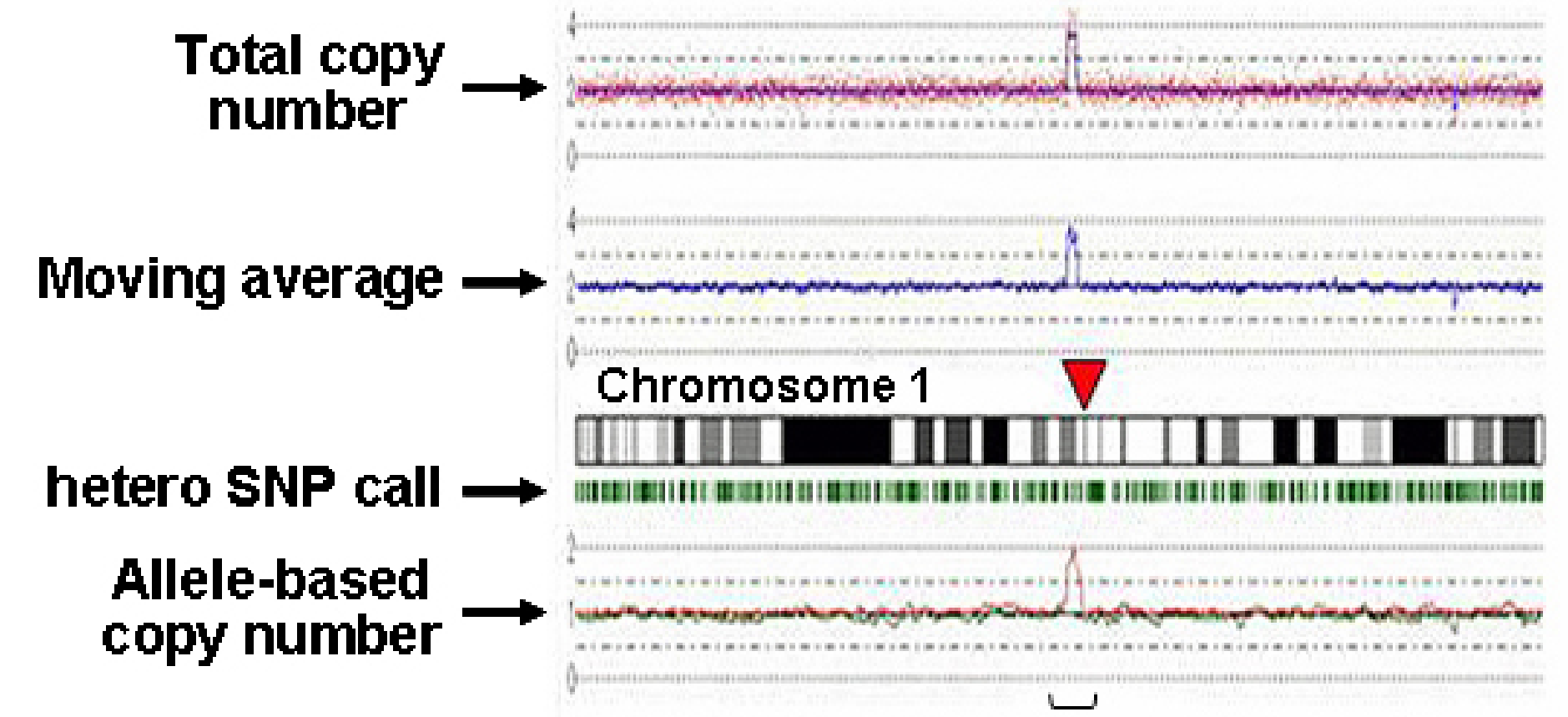

Allelic gain

B

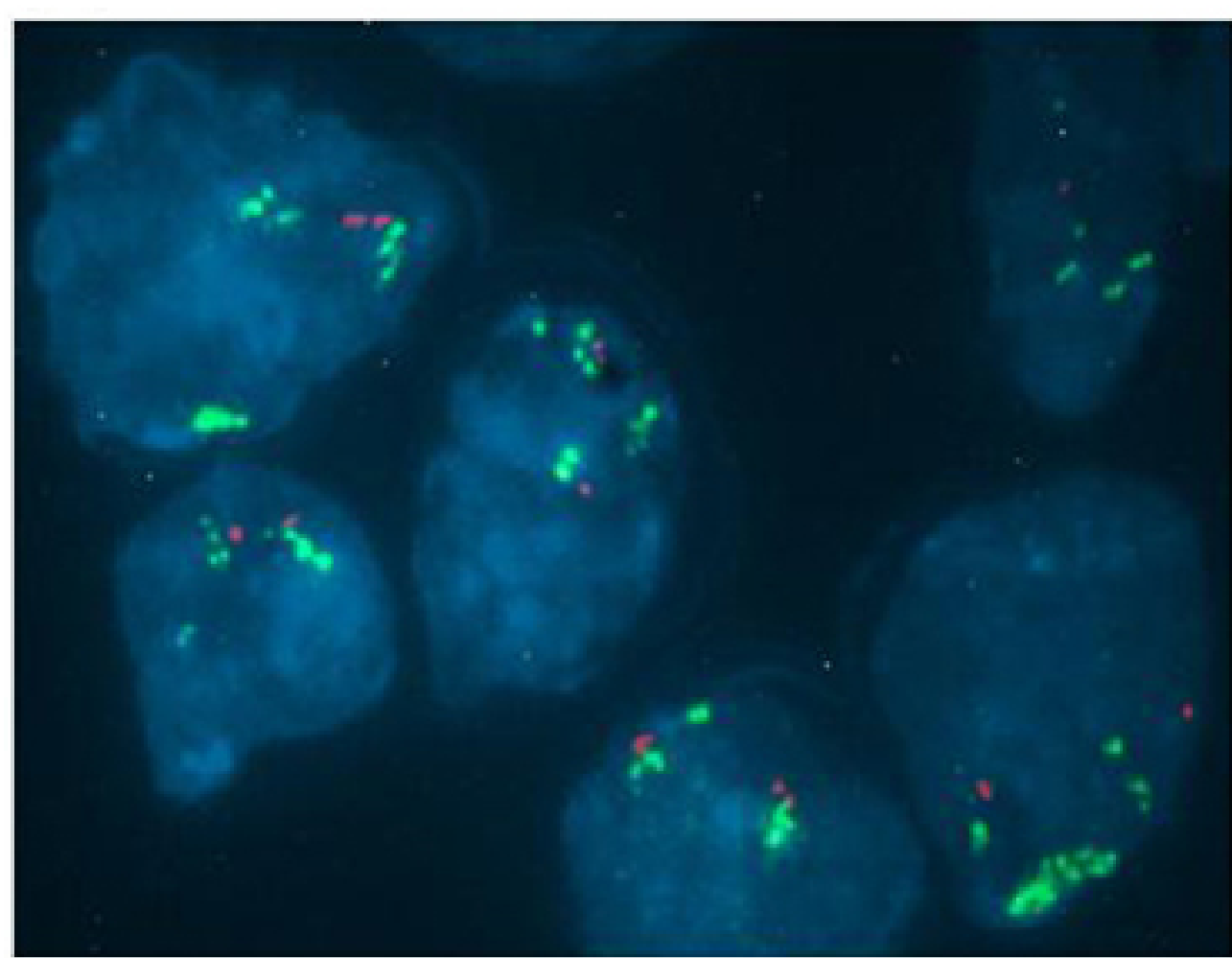

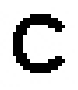

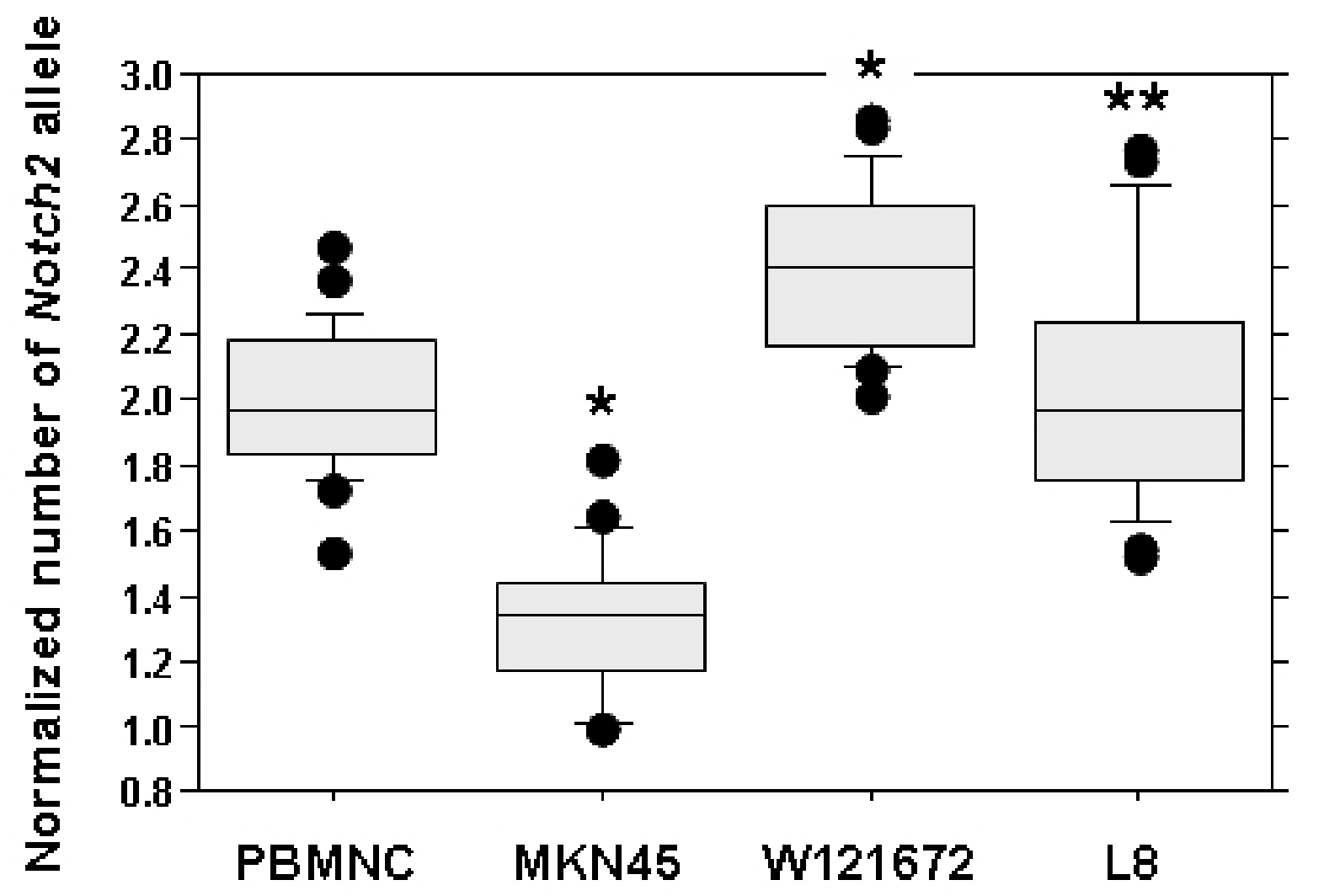


Fig. 4 (A)
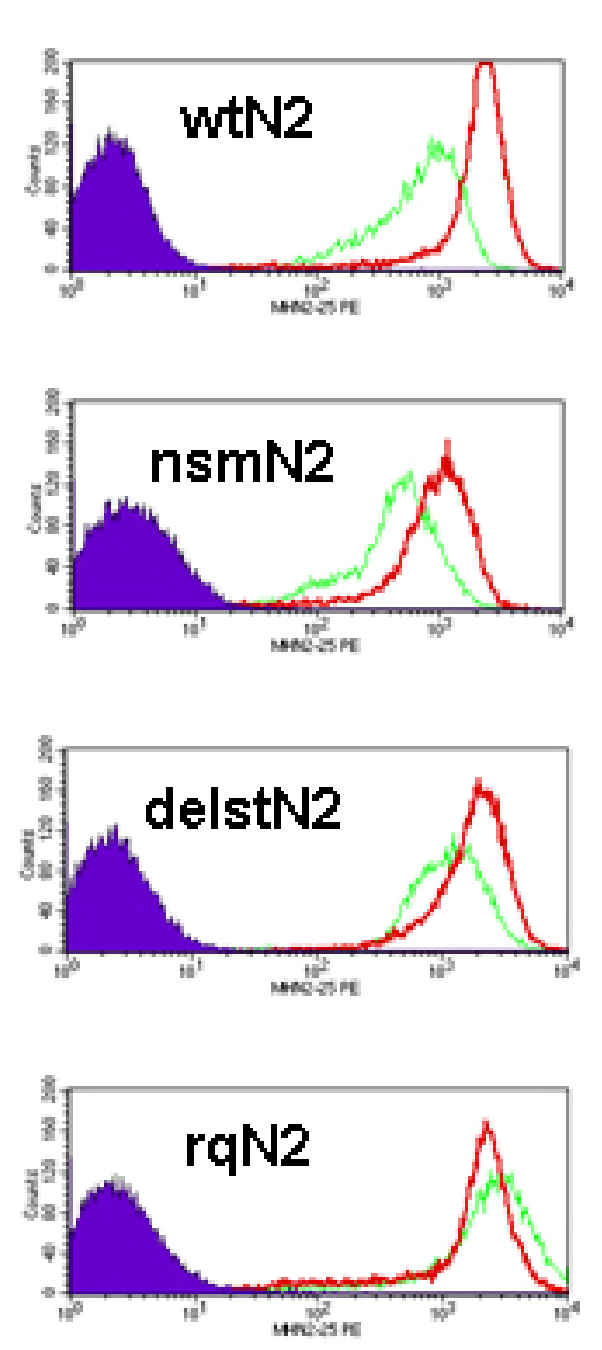

(C)

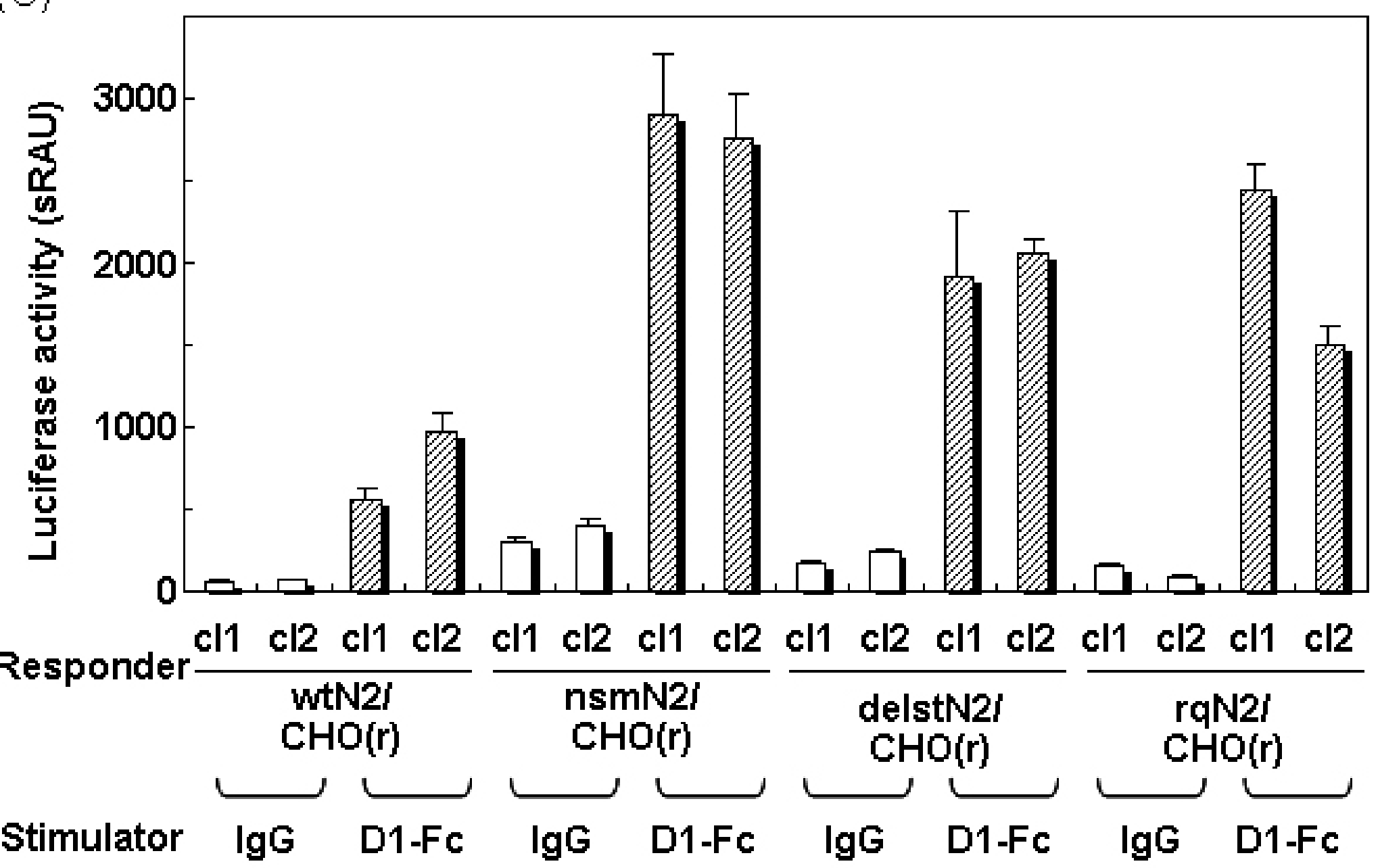

(B) wtN2I nsmN2I delstN2I rqN2I $\mathrm{CHO}(\mathrm{r}) \mathrm{CHO}(\mathrm{r})$ $\mathrm{CHO}(\mathrm{r}) \mathrm{CHO}(\mathrm{r}) \mathrm{CHO}$ MW ( $\mathrm{kDa})$

\section{5}

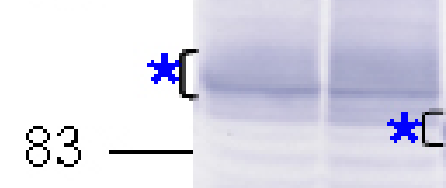

62

47.5

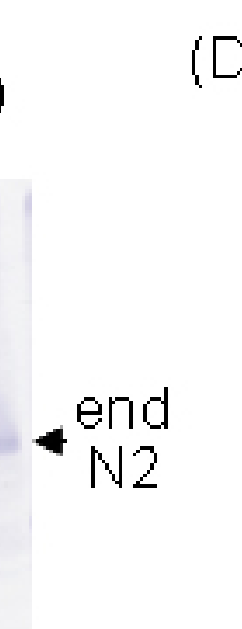

(D)

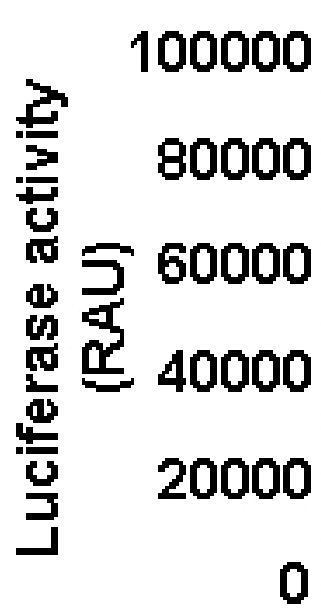$$
\text { . }
$$
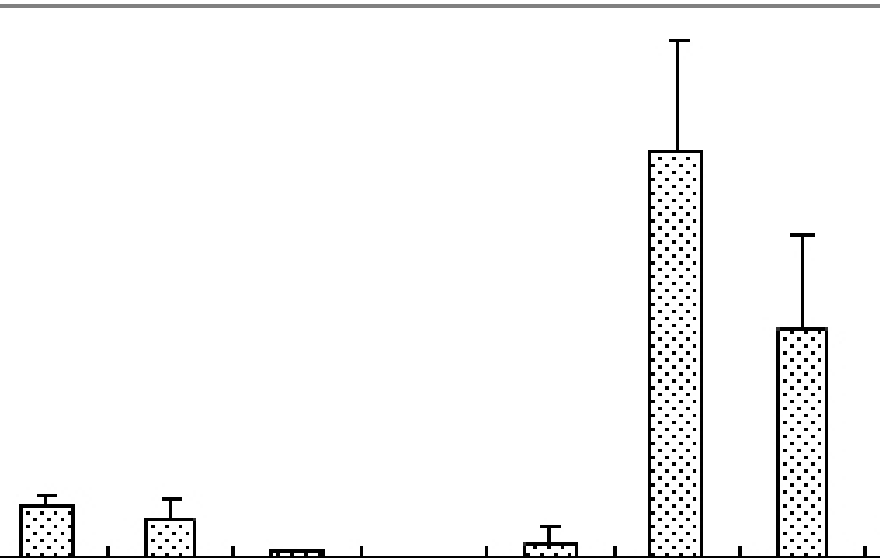

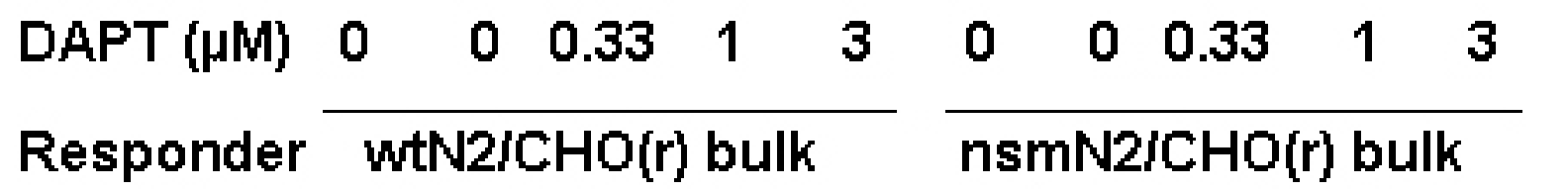

Stimulator $\overline{\lg G}$ 